Aus der chirurgischen Abteilung des St. Josephsstifts, Bremen. (Oberarzt: Dr. GroB).

\title{
Zur Chirurgie und Pathologie des Zwerchfells ${ }^{1}$ ).
}

Von Dr. Heintich Groß.

(Mit 3 Abbildungen.)

Die Chirurgie des Zwerchfells, wenn überhaupt von einer solchen als selbständigem Begriff die Rede sein kann (s. u.), wird repräsentiert durch die Operationen, welche ausgeführt wurden:

I. Zur Versorgung von Zwerchfellsverletzungen und Beseitigung ihrer Folgezustände (Eventration abdomineller Organe, Hernien);

2. bei vorher intaktem Zwerchfell zur Entfernung von Echinococcusblasen der Leberkonvexität oder Eröffnung subphrenischer Abszesse;

3. bei Tumoren der Brustwand, die sekundär das Zwerchfell einbegriffen hatten;

4. bei gleichzeitiger Verletzung der Brust- und Bauchhöhle, um von ersterer aus freien Zugang zu den subdiaphragmatisch gelegenen, ebenfalls verletzten Organen zu gewinnen (,,transpleurale Laparatomie").

Die Aufstellung dieser vier Gruppen wird in der Wahl ihrer Reihenfolge zugleich der historischen Entwicklung der Zwerchfellschirurgie gerecht.

Wenn A m burge ${ }^{2}$ ) in einem Überblick über die bisherigen Erfahrungen nur die erste bis dritte Rubrik berücksichtigt und ausfüllt, dagegen die vierte nicht aufstellt und die in sie entfallenden Betrachtungen überhaupt nicht erwähnt oder in der

I) Nach einem Vortrag mit Demonstration auf der VI. Tagung der Vereinigung Nordwestdeutscher Chirurgen, I2. XI. I9IO.

2) A m burger, Die operative Behandlung der Brustwand- und Mediastınalgeschwulste. Beitrage zur klin. Chirurgie I901, Bd. 30.

Deusche Zenschrift f. Chrurgie. 109 Bd 
ersten Gruppe registriert, so überschätzt er m. E. die bedeutsame Stellung der transpleuralen Laparotomie für den Ausbau des, wie er sagt, ,jungen Kapitels der Chirurgie“".

Die zielbewußte Ausführung der besonders von italienischen Chirurgen auf Postemsky's Empfehlung angewandten Methode mit weiter Eröffnung des Zwerchfells, um einen ungehinderten Ausblick auf die verletzten, subphrenisch gelagerten Organe (Milz, Magen, Leber) mit ausreichendem Operationsterrain zu schaffen, kennzeichnet den Fortschritt vielleicht mehr, als die resezierenden Operationen, deren Notwendigkeit sich bei der Exstirpation eines das Diaphragma erreichenden oder auch durchsetzenden thorakalen Tumors ergab.

Sind es auch nur imaginäre Vorstellungen, welche die Herstellung einer weiten Kommunikation zwischen den beiden großen Körperhöhlen als bedenklich erscheinen lassen, sie bestehen oder, wie wir in Anerkennung des Fortschritts sagen müssen, sie bestanden, und als ihr Ausfluß die Furcht, durch ausgiebige Incisionen der Zwerchfellsfläche verhängnisvolle Störungen des „mechanischen Gleichgewichts" (Gerulanos) ${ }^{1}$ ) hervorzurufen, unter dessen Schutz Brust- und Bauchorgane funktionieren.

Wie hoch s. Zt. die Leistungen des positiven Bauchdruckes und des negativen im Brustraum normiert wurden, zeigt z. B. noch die Arbeit B a r k e r's ${ }^{2}$ ) aus den 8oer Jahren: er addierte die Wirkungen der beiden Kräfte zu e i n e r, die geradezu mit einer gewissen Verve die Eingeweide des Abdomens bei Verletzungen des Zwerchfells in den Bereich des Thorax ziehe!

Von demselben Standpunkte der Beurteilung aus gelangte Beale ${ }^{3}$ ) zur Überschätzung seiner Beobachtung als physiologisch nicht oder schwer erklärbarer Kuriosität: ein Trauma hatte ein Stück Lunge durch den Zwerchfellriß in die Bauchhöhle treten lassen, statt, wie es seiner Ansicht nach hätte sein müssen, umgekehrt ein Bauchorgan in den Brustraum! Die Literatur verzeichnet mehrere ganz analoge Beobachtungen; ihre sehr einfache

I) Gerulanos. Deutsche Ze1tschr. f. Chirurgie Bd. 49.

2) Barker, Transactions of the pathol. soc. I $877, \mathrm{Bd} .28$.

3) Beale, On a case of hernia of lung through the diaphragme. Lancet I 882 . 
Erklärung finden sie darin, daß im Moment der Verletzung die Lunge sich in maximaler Inspirationsstellung befand; das Lungengewebe quoll aus der gesetzten Lücke des Zwerchfells heraus und wurde hier festgehalten.

,Wenn die Verletzung mit Sicherheit vorauszusehen ist, wird es vorteilhaft sein, sie nicht zu machen (de s'abstiner) und sich nicht zu einer Operation drängen zu lassen, deren Ausdehnung und Folgen man nicht übersehen kann."

In diesem Schlußsatz seiner Mitteilung von operiertem Osteosarkom der rechten Brustwand gab noch I886 $\mathrm{Humbert}^{1}$ ) seinem Bedenken gegenüber einer komplizierenden Zwerchfellverletzung Ausdruck, obwohl er den Erfolg seines Falles für sich hatte, die bei der Exstirpation der Geschwulst unbemerkt hergestellte Zwerchfellwunde von $7 \mathrm{~cm}$ Länge und $2 \mathrm{~cm}$ Breite durch die Naht schließen konnte und Heilung erzielte.

Wenn er dann auch, in den folgenden Worten einlenkend, seinen Rat, eine Operation, deren Fertigstellung eine Durchtrennung des Zwerchfells erfordert, unvollendet zu lassen und bei sekundär das Diaphragma affizierenden Tumoren sich mit einer Partialoperation zu begnügen, widerrufen möchte, sein Schlußsatz bleibt bestehen und mit ihm der deutlichst bekundete Ausdruck der Besorgnis vor unberechenbaren Gefahren, die aus der Eröffnung des $Z$ werchfells beim Operieren im unteren Pleuraraum resultieren.

Sie als in der Einbildung beruhend hinzustellen, erlauben die Erfahrungen bei den resezierenden Operationen und vor allem die weit großartigeren bei der Versorgung der Schuß- und Stichverletzungen des $Z$ werchfells, über die in den Zusammenstellungen von Lenormant ${ }^{2}$ ), Suter ${ }^{3}$ ), $W^{\prime}$ olf ${ }^{4}$ ) und Salomoni ${ }^{5}$ ) berichtet wird.

Letzterer stellt 229 operativ behandelte $Z$ werchfellverletzungen zusammen; I63 mal wurde lediglich auf thorakalem Wege vor-

I) $\mathrm{H} \mathrm{u} \mathrm{m} \mathrm{bert.} \mathrm{Revue} \mathrm{chir.} \mathrm{I} 886$.

2) Le normant. Revue de chir. I903.

3) Suter. Beiträge zur klin. Chirurgie 1905.

4) Wolf. Deutsche Zeitschr. f. Chirurgie igio.

5) Sa 10 moni. Clin. chir. I910 (Ref.). 
gegangen, $39 \mathrm{mal}$ per laparotomiam und $27 \mathrm{mal}$ per ,, Thorakolaparotomie" oder "transdiaphragmatischen Laparotomie" (Schaefer ${ }^{1}$ ).

Das Ergebnis - 36 Todesfälle - interessiert uns wesentlich nach der Richtung hin, daß, soweit ich mich orientieren konnte, einmal der Verschluß der Zwerchfellsöffnung, - sei sie durch das Trauma allein bedingt, oder bei der Operation erweitert, - durch die Naht ein definitiver (s, u.) war, und weiter irgend wie bedenkliche Konsequenzen (s. u.) einer weiten Kommunikation zwischen Bauch- und Brusthöhle sich nicht geltend machten; die Mißerfolge (vgl. F r e y ${ }^{2}$ ) sind jedenfalls nicht auf ihr Konto zu setzen.

Dieselbe Erfahrung zeitigten auch die sog. resezierenden Operationen. Als die umfangreichste Resektion im Zwerchfell galt bisher die v. Mikulic z(I89I ${ }^{3}$ ) mit Glück unter Excision eines handtellergroßen Stückes ausgeführte; außer ihr läßt die sorgfältige Sichtung des Materials durch A mburger nur noch die Betrachtungen Leisrink's ${ }^{4}$ ) und Hahn's ${ }^{5}$ ) gelten, wo gelegentlich der Exstirpation von Brustwandtumoren ein die ganze Dicke des Zwerchfells umfassendes Stück bis zum Umfang eines Fünfmarkstückes entfernt wurde.

Diesen drei wären aus der Kasuistik der neuesten Zeit noch zwei Fälle derselben Kategorie aus der russischen Literatur anzureihen: Gor ock ow und Derjushinsky $\mathrm{y}^{6}$ ) haben anscheinend in noch bedeutenderem Umfange, als v. Mikuli c $\mathrm{z}$, Resektionen am Zwerchfell vorgenommen; mit Unrecht, wie unser Überblick zeigt, erkennt letzterer Autor sich und seinem Landsmann den Ruhm zu, bis jetzt allein und zuerst die Operation ausgeführt zu haben.

Das Resumée aller dieser Erfahrungen geht dahin, daß das Ereignis der $Z$ werchfellseröffnung, auch wenn sie eine ganz weite offene Verbindung der beiden Körperhöhlen bedingt, wie sie die trans-

I) Schäfer, Beiträge zur klin. Chirurgie, Bd. 36 .

2) Frey. Wiener klin. Wochenschr. I 893 .

3) v. Mikulicz. Berl, klin. Wochenschr. 1891. (Ref.)

4) Leis rink. Archiv f. klin. Chırurgie 1889.

5) $\mathrm{H}$ a h n. Deutsche med. Wochenschr. 1888.

6) Derjushinski, Zweimalige Rippenresektion mit Entfernung eines bedeutenden Stuckes der Pleura und des Zwerchfells wegen eines primären Pleurasarkoms usw. Wiener klin. Wochenschr. 1905. 
pleurale Laparotomie erfordert, absolut nicht die Gefahren in sich birgt, die eine allzu ängstliche Beurteilung befürchten möchte; gerade ihr gegenüber, die in dem Ausfall des Tierexperiments (Kaninchen!) eine Stütze finden will, ist die Feststellung dieses Ergebnisses an der Hand eines Uberblickes über das vorliegende Material immer noch geboten.

Mit dem Nachweis ihrer Gefahrlosigkeit entfällt ein wesentlicher Grund, der berechtigt, den Eingriffen am Zwerchfell eine Sonderstellung in unserer großen Materie anzuweisen und sie zu dem selbständigen Kapitel der „Zwerchfellchirurgie“ zusammenzufassen.

Die Gesamtheit der Erfahrungen verweist sie in das größere Gebiet der ,Chirurgie im unteren Pleuraraum“, eine Bezeichnung, mit der sich ein weiterer passender Begriff umgrenzen ließe, der alle diese Operationen im Bereiche des unteren Thorax umfaßt, die bei Eröffnung des Cavum pleurae in der Vermeidung eines verhängnisvollen Pneumothorax ihr vornehmstes Ziel suchen. Der Einzelne wird indes weiter geneigt sein, die Operation am $Z$ werchfell mit einem besonderen Nimbus zu umgeben, so lange sich so selten Gelegenheit bietet, die lebenswichtigen Organe der beiden großen Körperhöhlen ohne trennende Scheidewand in einem Raum vereinigt $\mathrm{zu}$ sehen.

Möglich, daß die Bestrebungen zur Exstirpation des Cardiaund unteren Osophaguscarcinoms unter Schutz der Sauerbruch'schen Kammer oder des Bra u e r'schen Uberdruckverfahrens schon bald praktischere Ergebnisse zeitigen und damit dann für die $Z$ werchfelloperation ein weites Feld der Betätigung eröffnen; daß der mordlustige Stahl häufiger, als bisher, unter Durchquerung der Brusthöhle gegen die blutreiche Milz gezückt wird, ist wohl nicht $z u$ befürchten; da $B$ die anderweitigen Indikationen, die wir besprachen, künftig des Öfteren die Veranlassung zu operativen Maßnahmen am Zwerchfell böten, erscheint ebenfalls nicht wahrscheinlich, noch weniger, daß sich Betrachtungen, wie die folgenden, häufiger wiederholen, die - für sich eine ganz neue, also fünfte Gruppe der ,Zwerchfellchirurgie", und zwar in korrektester Präzision dieses Begriffes, vertretend - die chirurgische Be-

I) Frey. Wiener klin. Wochenschr. I 895. 
handlung von im Zwerchfell selbst lokalisierten Affektionen veranschaulichen.

Abgesehen von Anomalien in der ersten Anlage und Entwicklung, von Störungen in der Funktion, sekundärer Geschwulstentwicklung im Muskel ( $\mathrm{R} \mathrm{a} \mathrm{j} \mathrm{e} \mathrm{w} \mathrm{s} \mathrm{k} \mathrm{i}{ }^{1}$ ) usw. sind sonst trotz seiner enormen Flächenausdehnung und funktionellen Leistung krankhafte Zustände am Zwerchfell überhaupt kaum bekannt.

Eigentümliche degenerative Veränderungen mit fettiger Entartung und Atrophie des Zwerchfells, die zuerst von $\mathrm{C}$ a 11 e $\mathrm{n} \mathrm{d} \mathrm{e} \mathrm{r}{ }^{2}$ ) und $\mathrm{Marsh}{ }^{3}$ ), dann von $\mathrm{Z}$ a h ${ }^{4}$ ) festgestellt wurden, erhalten wohl erst durch die Koinzidenz mit analogen des Myokards ihre besondere pathologische Bedeutung.

Als weitere Illustrationen zur "Pathologie“ des Zwerchfells finden sich sonst in der Literatur nur zwei Cysten aufgeführt, Sektionsbefunde, die von $L$ archer ${ }^{5}$ ) und $L a b$ o u 1 èr $e^{6}$ ) beschrieben wurden. Die Deutung ihrer diaphragmatischen Genese ist indes etwas problematisch, wie mir scheint, durch die Würdigung der topographischen Lageverhältnisse allzusehr beeinflußt.

Als besonderen Zufall habe ich deshalb zu betrachten, da $B$ sich mir Gelegenheit bot, in drei Fällen eine ,chirurgische" Affektion des Zwerchfells zu beobachten und operativ zu behandeln. Zweimal handelte es sich um zirkumskripte Tuberkulose des muskulären Teils, im dritten Fall um eine große sarkomatöse Neubildung.

F a 11 I. Sch., Julius, 6 Jahre alt, Kapitänssohn. Aufgenommen I2. VI. Igo6. Bei dem bisher gesunden, wenngleich schwächlichen Jungen soll sich angeblich vor I4 Tagen plötzlich unter Schmerz eine Geschwulst der rechten Brustwand gezeigt haben, so daß der Hausarzt erst an eine Lungenhernie dachte.

Untersuchung ergibt zwischen vorderer und hinterer Axillarlinie zwischen 8. und 9. Rippe eine halbhühnereigroße, undeutlich fluktuierende Anschwellung bei verschiebbarer, nicht geröteter Haut. Über der rechten Lunge, hesonders Unter- und Mittellappen, vereinzelte Rhonchi. Diagnose: Caries costarum.

I) Ra jewski. Virchows Archiv Bd. 66.

2) Ca 11 e nder. Lancet i 867 .

3) M a rsh. eod. loc.

4) Z a h n. V. A.

5) L a rcher. Arch. génér. I 868.

6) L a b o ul ère. Gaz. méd. de Paris is 3 . 
Operation I4. VI. Bogenschnitt legt in der Brustwandmuskulatur zwischen der IX. u. X. Rippe gelegene käsig-sulzige Masse frei, die der verdickten Pleura parietalis fest aufsitzt. Nach ausgiebiger Resektion der Rippen wird Pleurahöhle eröffnet. Lunge diffus zart adhärent. Nach Incision des Brustwandherdes zeigt sich, daß von seinem hinteren Pol aus ein käsiger Gang sich im Brustraum fortsetzt. Unter Kontrolle des Auges wird er im gewundenen Wege bis aufs Zwerchfell verfolgt. Hier nach Ablösung der ebenfalls adhärenten Lunge zeigt sich eine graugelbliche, käsige fünfmarkstückgroße Platte, die das Zwerchfell durchsetzt. Exkochleation und Exstirpation der Randpartien mit der Schere. Leber, sowie freiliegende vordere Bauchwand sind nach oben durch zarte, sicher ganz frische strang- und flächenhafte Adhäsion abgeschlossen.

Vernähung des ovalär gestalteten Zwerchfellsloches durch 5 Katgutsuturen. Äußere Wunde zum Teil vernäht; Drainage.

Mikroskopische Untersuchung der käsigen Massen ergibt exquisite Tuberkulose.

2I. VI. Fieber von $39,5^{\circ}$ abends. Rechts obere Bauchgegend über Leber besonders erscheint aufgetrieben. Druckempfindlichkeit. Gutes Verhalten der Brustwunde (Verbandwechsel).

27. VI. Unter feuchtem Verband sind Erscheinungen etwas zurückgegangen. Temperatur $38^{\circ} \mathrm{C}$.

2. VII. Heute im Stuhl deutlich Eiter, auch mikroskopisch festgestellt. Seitdem glatter, afebriler Verlauf.

I7. VII. In Heilung entlassen. Vorstellung des Patienten I2. XI. I910. Gute körperliche Entwicklung. Lungenbefund negativ. Unregelmäßige Narbe von $20 \mathrm{~cm}$ Länge ist ziemlich tief bis aufs Zwerchfell eingezogen; Rippendefekt von 9- $10 \mathrm{~cm}$ Länge. Sinus diaphragmaticus gänzlich verödet.

Über die Genese des tuberkulösen $Z$ werchfellherdes werde ich nach Mitteilung des zweiten Falles mich äußern.

Eine Erörterung verdient die Entwicklung des Bauchabszesses, der in den Darm perforierte und diesen mit dem Stuhlgang verließ.

Daß ein solcher Vorgang sich abgespielt hat, erscheint durch den Nachweis des Eiters im Stuhlgang, gleichzeitig mit dem Schwinden der klinischen Erscheinungen, außer Zweifel. Die Frage ist nur, welcher Natur die Eiterung war, die zur Auftreibung der rechten oberen Bauchseite führte.

Ich glaubte, die Annahme eines tuberkulösen Abszesses, der zufällig gerade nach der Operation, ohne früher irgendwelche Er- 
scheinungen gemacht zu haben, den Weg in den Darm fand, $a b-$ lehnen zu müssen.

Gegen sie spricht auch, daß der tuberkulöse Herd des Zwerchfells nach der Bauchhöhle zu durch allerdings zarte Adhäsionen geschützt war, und bei ihrer Lösung keine Spur eines Abszesses festgestelllt wurde; die Leber lag in spiegelnder Oberfläche frei vor.

Für die große Wahrscheinlichkeit einer a k u t e n Infektion, die also auf Kosten derOperation zu setzen wäre, sprechen Temperatursteigerung und die damit einsetzende Schwellung der oberen Bauchgegend.

Auffallend und schwer erklärbar bliebe dann nur die Schnelligkeit, mit der es dem Eiter gelang, den Weg über die Leber hinweg ins Darminnere zu finden, ohne zu einem subphrenischen Abszeß zu führen.

Mein zweiter Fall bedingte Vorgehen von der Bauchseite her.

F a 11 2. Joseph F., 33 Jahre alt, Fleischbeschauer, L. (Oldenbg.). Aufgenommen 5. V. IgIo.

Anamnese: Seit Juli Igog „Bauchfellentzündung“, die langsam besser wurde; seit to Wochen tritt unter rechten Rippenbogen eine in letzter Zeit besonders zunehmende Anschwellung hervor.

Befund: Blasser, graziler Mann, Puls beschleunigt. Herz und Lunge o. Bfd. Im oberen Gebiet des rechten Rectus, bis an Rippenbogen reichend, medial über Linea alba, nach unten bis Nabelhöhle, liegt faustgroßer, deutlich fluktuiérender Tumor mit geröteter Haut. Leib sonst weich; im Epigastrium Dämpfung und Empfindlichkeit.

Diagnose: In Perforation begriffener Bauchdeckenabszeß, wahrscheinlich von Leber bzw. Gallenblase ausgehend.

Sofort Operation. Längsschnitt eröffnet fast $3 / 4$ l fassende Abszeßhöhle, zum Teil in den Bauchdecken gelegen; ein Loch im Peritoneum führt in einen Raum, der nach oben von Leber, nach unten von verwachsenen Darmschlingen begrenzt ist. Tamponade, Drainage.

Kult. Untersuchung des Eiters negativ, Überimpfung auf Tier ergibt Tuberkulose (Hygienisches Institut: Dr. M e ye r).

30. V. Nachdem Wundhöhle sich bis auf $2 \mathrm{~cm}$ langen Spalt geschlossen, von dem aus Sonde weit in die Bauchhöhle vordringt, zweite Operation.

Umschneidung der äußeren Fistelmündung, Verfolgen des Ganges, der erst gerade in die Tiefe geht, dann ebenso steil nach vorn oben unter den Rippenbogen umbiegt; nacheinander werden ro. bis 7 . Rippe partiell reseziert, um exquisit tuberkulöse, von käsigen Granulationen ausgekleidete, etwa $2-3 \mathrm{~cm}$ breite Bahn freizulegen, die, etwa in Mammillarlinie beginnend, im schrägen Verlauf in der Richtung nach der 
linken Artic. sternoclav. zwischen Zwerchfell und Leberoberfläche sich nach oben bis zum Zwerchfell zieht. In diesem, $3 \mathrm{~cm}$ weit von der 7. Rippe entfernt, liegt ein fingerabdruckartiger, scharfrandiger Herd von $3 \mathrm{~cm}$ Länge. In der weiteren Umgebung ist Leberserosa mit vielen Adhäsionen bedeckt und stark verdickt. Exstirpation des Herdes unter Resektion des Zwerchfells in gut $\mathrm{I} \mathrm{cm}$ Peripherie, dabei Eröffnung der Pleurahöhle; Pneumothorax wird durch sofortige Tamponade, die bereitgehalten war, sehr gering gestaltet.

Nach Besorgung Wundhöhle offene Wundbehandlung.

Mikroskopische Untersuchung (Prof. B orrmann) des Zwerchfellherdes: knötchenförmige Tuberkulose mit Riesenzellen.

24. VI. Gute Heilung. Unter der linken Clavikula wird eine nußgroße tuberkulöse Lymphdrüse entfernt (Schle ich).

I7. VII. In Heilung bei gutem Allgemeinzustand entlassen. Lungenbefund negativ.

I2. XI. Demonstration: Patient hat 24 Pfund an Körpergewicht zugenommen, sieht blühend aus. Wunde gut vernarbt, etwa $20 \mathrm{~cm}$ lang, $8 \mathrm{~cm}$ breit, zeigt am Außenrand noch einen kleinen oberflächlichen Defekt.

Der klinische Bericht, so bemerkenswert auch der Senkungsvorgang der tuberkulösen Eiterung sich gestaltete, bedarf keines weiteren Kommentars.

Gehen wir zur genetischen Beurteilung dieser beiden Fälle von Zwerchfelltuberkulose über, so hat die mikroskopische Untersuchung keine Anhaltspunkte ergeben.

Die primäre Muskeltuberkulose ist so selten, da $B$ ich hier einer dahingehenden Annahme nicht das Wort geben möchte.

Vielmehr erscheint es wahrscheinlicher, daß die tuberkulöse Infektion des $Z$ werchfells von einem zirkumskripten pleuralen; bezw. peritonealen Herd aus erfolgte, oder daß eine tuberkulöse Lymphdrüse in nächster Umgebung der Serosa von ihr aus die Muskulatur des Diaphragmas infizierte.

In technischer Hinsicht boten beide Fälle keine großen Schwierigkeiten, speziell ersterer nicht, da überall in der Umgebung des Herdes pleurale Adhäsionen bestanden, und es sich um relativ kleine Defekte des $Z$ werchfells handelte, deren Versorgung im ersten Fall durch wenige Nähte sich leicht herstellen ließ, und im zweiten der Tamponade überlassen werden konnte, da die Leber die Brusthöhle abschloß, und die Gefahr des Prolapses abdomineller Organe nicht vorlag. 
Weit größere technische Schwierigkeiten bot der dritte Fall meiner Kasuistik; er liegt mehrere Jahre zurück und stammt noch aus meiner Jenenser Zeit.

Die Exstirpation des gut kindskopfgroßen, das Zwerchfell durchsetzenden Sarkoms, das zudem in Lunge, Herzbeutel und Milz eingedrungen war, bedingte die $\mathrm{umfangreichste}$ Resektion, die jemals vorgenommen wurde.

Es resultierte ein fast mannskopfgroßer Defekt (= ein Sechstel bis ein Fünftel des Zwerchfells), dessen Verschluß sich jedoch ohne weitere plastische Operation lediglich unter Zuhilfenahme ausgiebiger Resektion der Brustwand, speziell seines Rippenanteils, ganz exakt durch die Naht ermöglichen ließ.

F a 11 3. H., Hermann, 30 Jahre alt, Landbriefträger aus K. Aufgenommen 3. XI., entlassen 22. XI. Ig02.

Im Beginn des Sommers traten bei dem bisher gesunden, wenn auch stets schwächlichen Patienten erst Kreuzschmerzen mit linksseitigem Seitenstechen auf, dann die Erscheinungen eines hartnäckigen, jedoch leichten Lungenkatarrhs. Vor etwa 2 Monaten bemerkte er an der linken unteren Brustwand eine kleine Verhärtung, die, ohne ernstliche Beschwerden zu verursachen, sich zu einer Anschwellung ausbildete.

Nachdem der Kranke bis vor 3 Tagen seinen schweren Dienst vollständig hatte versehen können, entschloß er sich schließlich, durch das Wachstum der Brustwandgeschwulst, sowie stärkeren Hustenreiz mit Auswurf und Bruststechen beunruhigt, dem Rat seines Arztes Folge zu leisten und die Klinik aufzusuchen.

Aufgenommen 3. XI. Igo2. Temperatur 37,7. Beide Fossae supraclaviculares des hageren, langaufgeschossenen, blassen Mannes sind eingesunken, besonders die rechte; die Untersuchung in der medizinischen Poliklinik (Prof. M a $\mathrm{t} t \mathrm{~h}$ e s) ergibt in Bestätigung des von uns erhobenen Befundes: diffuse trockene Rasselgeräusche über beiden Lungen bei abgeschwächtem Atemgeräusch.

$\mathrm{Da}$ außerdem bei dem Kranken seit längerer Zeit Nachtschweiße bestanden, worauf das Begleitschreiben des behandelnden Arztes besonders aufmerksam machte, wurde auch unserseits, in Übereinstimmung mit der Diagnose des Arztes, Phthisis pulm. als wahrscheinlich bestehend angenommen, und die gleichmäßig geformte, weiche, nicht schmerzhafte Vorwölbung der linken unteren Brustwand als Rippentuberkulose angesprochen:

Etwa $6 \mathrm{~cm}$ lang, $3 \mathrm{~cm}$ breit, ist sie im Bereich der 8. und 9. Rippe, zwischen vorderer und mittlerer Axillarlinie, gelegen und überragt in ihrer höchsten Prominenz das Niveau der Umgebung um $3 / 4-\mathrm{I} \mathrm{cm}$. 
Die bedeckende Haut ist gut verschieblich, nicht gerötet, der Tumor selbst absolut fest auf den Rippen fixiert und bei Druck leicht schmerzhaft.

Patient, abends aufgenommen, dringt als Selbstzahler zur Operation, die am nächsten Morgen vorgenommen wird.

Operation 4. XI. Bogenförmiger Schnitt auf dem palpablen freien unteren Rand der 9. Rippe, legt zuerst die untere Fläche des Tumors frei, der nach Durchtrennung der bedeckenden dünnen Muskelschicht in etwa $5 \mathrm{~cm}$ Länge sich darbietet, dann, unter weiterer Ablösung der deckenden Schicht, seine ganze Außenfläche.

Der Tumor tritt zwischen 8. und 9. Rippe, die etwas auseinandergewichen sind, hervor; seine weißliche Farbe und feste Konsistenz fallen auf; die Incision ergibt Geschwulst.

Die Pleura costalis an den beiden Seitenpolen des Tumors beweist durch ihre Trübung und Undurchsichtigkeit das Vorhandensein von Adhäsionen. Ein Einschnitt zeigt, daß die Pleura mit der Oberfläche des Tumors verwachsen ist, und überzeugt zugleich, daß dieser im Pleuraraum sich verbreitert.

Verlängerung des Weichteilschnittes nach vorn bis zum Rippenknorpel, nach hinten bis jenseits der Skapularlinie. In derselben Länge wurde 8.- Io. Rippe subperiostal reseziert. Beim Ablösen der hinteren Periostfläche der 8 . und 9. Rippe brechen diese entsprechend der Mitte der Geschwulst ein, nachdem sie sich an der Vorderfläche leicht von dem überstehenden Tumor hatten isolieren lassen; sie sind entzündlich erweicht, die 9. Rippe zeigt an ihrem oberen Rand einen kleinen Randdefekt (Drucknekrose).

In fortschreitender Erweiterung des zuerst angelegten Schnittes durch die Pleura costalis wird die Lateralfläche des Tumors nach und nach entwickelt.

Der zwischen 8. und 9. Rippe hervorgetretene Teil entspricht dem Übergang von der lateralen zur hinteren Seitenfläche. Von ihm aus gelangt man unter stumpfer Lösung ziemlich fester Adhäsionen auf die Oberfläche der Geschwulst, die nach dem Mediastinum zu schräg aufsteigt; ihr liegt mit verdickter, adhärenter Pleurabedeckung die Lungenbasis auf, die nach oben und etwas nach innen gedrängt ist. Pleura costalis und pulmonalis sind fest verwachsen.

Die Lunge wird bis auf eine gut handtellergroße Fläche abgelöst, bis, etwa der Mammillarlinie entsprechend, in das Parenchym eingewachsene Tumormasse zur Resektion einer $8 \mathrm{~cm}$ langen, an seiner, nach dem Mediastinum zu gelegenen, Basis $4 \frac{1}{2} \mathrm{~cm}$ hohen und auf der Fläche etwa $6 \mathrm{~cm}$ breiten Keils aus der Lunge zwingt. Unterbindung der spritzenden Gefäße; U̇bernähung.

Die laterale Fläche des Herzbeutels liegt vor; auch in ihn ist der Tumor eingedrungen, so daß eine Resektion des Perikards etwa im Umfang eines Fünfmarkstücks notwendig ist. Naht der Offnung, neben 
deren vorderem Rand der N. phrenicus durchscheint, gelingt über dem freiliegenden Herzen ohne Spannung.

Die weitere Isolierung des jetzt in ganzer Oberfläche vorliegenden Tumors auch der vorderen und hinteren Seitenfläche geht glatt vor sich; vorn ist er mit der Pleura der Brustwand verwachsen; die Freilegung der hinteren Seite erfolgt nach Trennung einiger festerer, breiter Stränge, die nach dem Mediastinum, bzw. hinterer Brustwand führen.

Schließlich liegt die ganze Basis der Geschwulst übersichtlich frei, dem Zwerchfell mit ziemlich steil abfallenden Rändern in scharfer Begrenzung aufliegend. Mit ihrem oberen medianen Pol, dem höchsten Punkt, der etwa der Verbindung der 5. Rippe mit dem Sternum entspricht, ragt die Geschwulst eben in das Zentrum tendineum ein, sonst gehört sie ganz der Muskelplatte des linken $Z$ werchfells an, dessen normale Lage (Wölbung) erhalten ist.

Da Tumor nicht zu lösen, wird das Zwerchfell in $2 \mathrm{~cm}$, stellenweise $3 \mathrm{~cm}$ weiter Peripherie durchschnitten, zuerst an der vorderen medianen Seitenfläche. Die Geschwulst ragt hier frei ins Abdomen vor und erstreckt sich mit ihrem am weitesten median vorspringenden Teil auf die Leberoberfläche in der Gegend der Porta fort, hier locker verwachsen; weiter nach hinten liegt sie dem linken Leberlappen, dann, - getrennt nur von diesem durch einen flächenhaft adhärenten Netzzipfel, der reseziert wird, - dem Magen auf.

Die Umschneidung der lateralen Zwerchfellsbegrenzung gestaltet sich dadurch schwieriger, daß der Sinus diaphragmaticus durch ziemlich feste und dichte Verwachsungen seiner Wände unterhalb des Tumors obliteriert ist; er wird unter Erhaltung der entzündlich verdickten Pleura wiederhergestellt.

Nach der Durchtrennung des Zwerchfells hier, die damit im ganzen Umkreis des Tumors vorgenommen ist, zeigt sich, daß letzterer in den oberen Milzpol eingedrungen ist und durch resezierende Schnitte entfernt werden muß. Übernähung der blutenden Milzfläche.

Entnahme der Geschwulst. Es liegt ein bequem für einen kräftig entwickelten Mannskopf durchgängiges Loch im Zwerchfell vor, von vorn abgerundeter, hinten nach dem Mediastinum zu spitzwinkliger Form. Durch einige Scherenschnitte an der vorderen Begrenzung wird ein regelmäßiges Oval hergestellt.

An Stelle der Gazeschicht, die bisher die abdominellen Organe (Magen, Colon transversum, Leber) bedeckte, wird zum Schutz über diese das große Netz emporgeschlagen und über den Magen weg mit dem lateralen Wundrand des Zwerchfells vernäht. Daruber wird die Naht des Zwerchfells ausgeführt (in toto I6 Suturen); nachdem zuerst die Wundränder in der Mitte unter einiger Spannung mit besonders starken Katgutfäden aneinandergelegt sind, gelingt die Vereinigung der übrigen Wundfläche unter Einsenkung der äußeren Brustwand üherraschend leicht. 
Excision der Pleura costalis mit Rippenperiost und der aufliegenden Thoraxmuskulatur im Bereich des ganzen Operationsterrains. Darüber Hautnaht; im vorderen Wundwinkel gazeumwickeltes Drain.

Am nächsten Tag bei quälendem Hustenreiz abends $39,5^{\circ}$, dann bis zum fünften, wo Tampon entfernt wird, fieberfreier Verlauf bei zunehmendem Husten mit Auswurf. Linke Seite bleibt beim Atem sichtbar zurück, Atmungsfrequenz 25-30.

Vom 9. XI., also 5 Tage nach Operation, bis I6. XI. inklusive Pneumonie des linken Unterlappens in ausgesprochenem Bilde. Mehrfach wurden Diplokokken im Sputum nachgewiesen. Temperatur 38,6, Puls IOo-I20.

Am I4. Tage wird, da Retention vermutet, äußere Naht entfernt und Wundhöhle wieder eröffnet, dabei Zwerchfellnaht in ganzer Länge übersichtlich freigelegt; sie hatte standgehalten. Die linke Lungenbasis fühlt sich derb an. Resektionsfläche, wie die ganze Wundhöhle fibrinös-eitrig belegt.

Vom I6. XI. an schneller Heilungsverlauf; vom 22. XI. kann Patient bereits in poliklinische Behandlung entlassen werden. Mitte Januar ist die Heilung vollendet. Im April nimmt Patient seinen Dienst wieder auf. - Tod im Juni d. J. in Kachexie unter Lähmungserscheinungen. Der betreffende Arzt, der mir diese Auskunft auf Anfrage gab, ließ unentschieden, ob Rezidiv oder Metastase, die also das Rückenmark betroffen haben würden, vorgelegen habe.

Beschreibung der exstirpierten Geschwulst (Fig. I): Die Geschwulst, von einer an Stellen der Adhäsionen dickeren, sonst zarten Kapsel eingehüllt, ist von knolligen Massen gebildet, deren Konturen auch auf den Durchschnittsflächen (vgl. Fig. 2) noch deutlich hervortreten.

Die Konsistenz ist fest, an den peripher vorspringenden Kanten etwas weicher, als im Zentrum.

Der größte Längendurchmesser der Geschwulst - in situ der Verbindungslinie der äußersten Vorwölbung der zwischen 8. und 9. Rippe vortretenden Seitenfläche (etwa mittlere Axillarlinie) und dem medianwärts, nach dem Mediastinum $\mathrm{zu}$, am weitesten vorspringenden Punkt beträgt I4,5 (Fig. I, p-q), der senkrecht $z u$ ihm verlaufende größte Breitendurchmesser (m-n) I3,8 cm.

Fig. 2 zeigt auf einem quer zum $Z$ werchfell angelegten $S$ chnitt die Dickenverhältnisse; der größte bei $a-b$ beträgt $7 \frac{1 / 2}{\mathrm{~cm}}$, die kleinsten $4^{1 / 2}$ bei $c-d, 5,3$ bei e-f; $g$ bezeichnet laterale, h mediale Seitenfläche, zugleich die Endpole des den Tumor durchsetzenden Zwerchfellmuskels, der sich deutlich auf der Schnittfläche markiert.

Die obere, thorakale Fläche ist in ibrem größeren lateralen und hinteren Teil ziemlich eben, wenn auch hier nach Entfernung der Einsenkungen ausfüllender Bindegewebsmassen die höckerige Beschaffenheit der Geschwulst deutlich hervortritt. 
Vorn und medianwärts markiert sich eine tiefere Einsenkung, von in ihrer Kuppe etwa Walnüssen an Größe entsprechenden Knollen eingefaßt; je 2, etwas kleinere, springen am medialen oberen Pol nach dem Herzen und der Lunge zu vor; sie waren in das Parenchym der letzteren, wie ins Perikard eingedrungen; die der Lunge entsprechenden sind auf Fig. I sichtbar ( $x y)$, umgeben vom Lungengewebe $(q-z)$.

Das Zwerchfell als Grenze des pleuralen und abdominalen Teiles der Geschwulst zeigt Fig. I (Resektionslinie des Zwerchfells bei a, a, a);

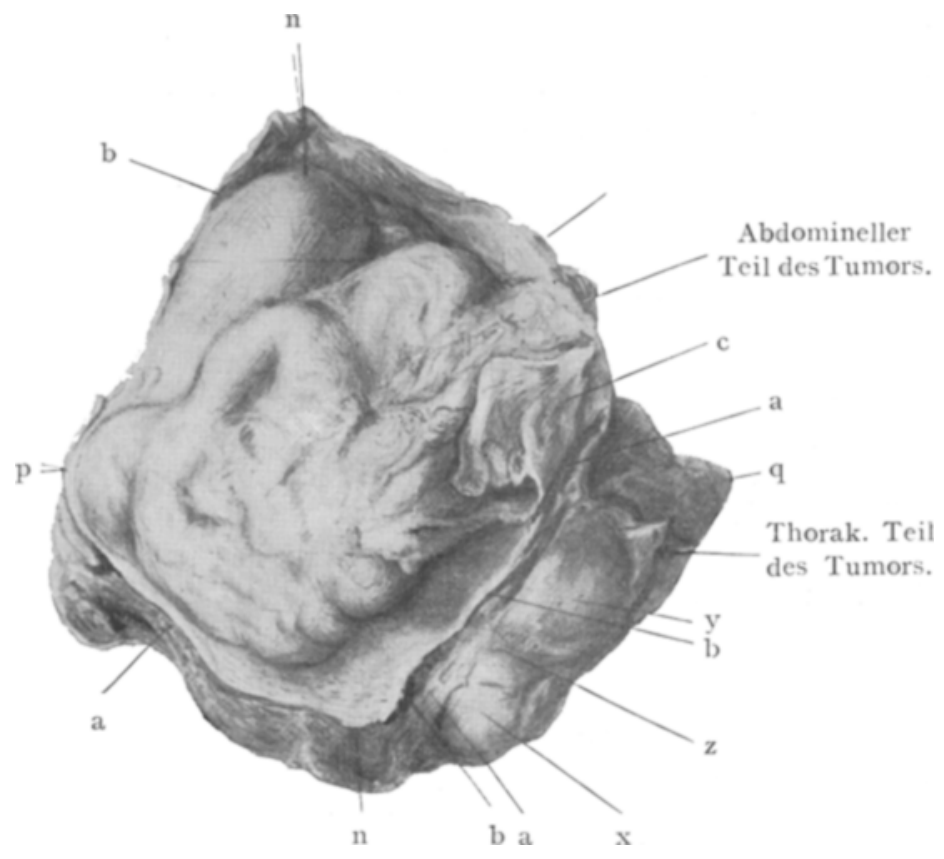

Fig. I.

bei $o$ ist noch das abgetrennte Milzgewebe vorhanden, bei $\mathrm{c}$ der abgetrennte Netzzipfel; bei b, b Peritoneum.

Der abdominelle Abschnitt des Tumors zeigt eine nicht unerhebliche Verjüngung; er springt scharf ins Zentrum der Zwerchfell-Resektionsfläche vor, etwa $3-4 \mathrm{~cm}$, ist $9^{1 / 2} \mathrm{~cm}$ lang und $6 \frac{1}{2} \mathrm{~cm}$ breit; seine Masse ist ausgesprochen knollig (vgl. Fig. I).

In Fig. 3 ist das Verhalten des Zwerchfells an der lateralen Fläche des Tumors veranschaulicht: Erst nach der Entnahme des Präparates, bei näherer Besichtigung, zeigte sich eine bei derOperation nicht festgestellte Diastase des Zwerchfells am lateralen Pol der Geschwulst: bei a, a treten die oberen Fasern hervor, bei $a_{2}, a_{2}$ die unteren, voneinander durch eine $3-4 \mathrm{~cm}$ dicke Geschwulstmasse getrennt. 
Das Auseinanderweichen der Muskelplatten beginnt etwa $3 \mathrm{~cm}$ vor der lateralen Seitenfläche der Geschwulst, und zwar ist es bedingt durch zwei intramuskuläre Knollen, die, wie auch in Fig. 2 noch er-

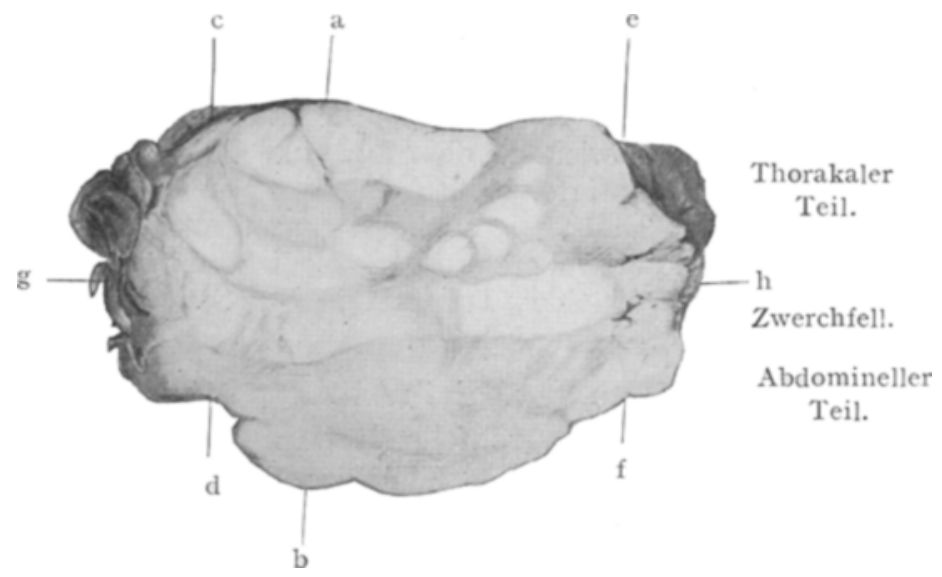

Fig. 2. Durchschnitt in der Längsrichtung des Tumors. Größe $2 / 3$. Auffallend ist hier die Integrität des Zwerchfells, das an anderen Stellen gänzlich substituiert ist.

kenntlich, den Muskel immer weiter auseinandergetrieben haben. Die beginnende Diastase ist auch in Fig. 2 bei $g$ (lat. Seitenfläche) zu sehen, jedoch auf diesem nahe dem größten Längsdurchmesser angelegten

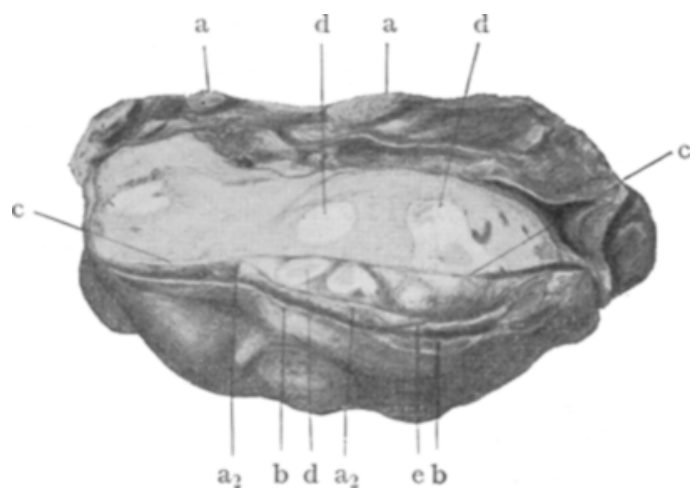

Fig. 3.

Schnitt mehr als Verbreiterung des die Tumormasse durchsetzenden Zwerchfelles erscheinend. Der Schnitt hat gerade Übergangsstellen der beiden Knollen getroffen, die, ein größerer bzw. längerer und ein kleinerer, auf der Abbildung 3 noch erkennbar sind.

$\mathrm{C} \mathrm{C}$ in Fig. 3 bedeutet die Verbindungslinie zweier senkrecht 
zueinander angelegter Schnitte, b, b die Ansatzstelle des Peritoneum diaphragmaticum, e ein Stück der den Tumor umhüllenden Kapsel.

Die auf der Schnittfläche bei d, d, d hervortretenden gelblichweißen, ovalen und rundlichen Massen entsprechen besonders gefäßarmen Partien; stellenweise sind hier kleinere nekrotische Herde eingelagert.

Sonst ergibt die mikroskopische Untersuchung ein Fibrosarkom, an dem besonders der Reichtum an Riesenzellen auffällt. Herr Prof. $\mathrm{R}$ is el, jetzt in Zwickau, damals Leipzig, dem ich die mikroskopischen Präparate zeigte, stellte eine weitgehende Übereinstimmung im histologischen Bilde, wie auch im ganzen anatomischen Verhalten mit Lymphdrüsensarkomen fest vom Mediastinum und linken Brustraum, von denen sich das Präparat in der Sammlung des Leipziger pathologischen Instituts befindet.

v. Horock ${ }^{1}$ ) aus der Klinik Alberts hat s. Zt. vorgeschlagen, größere Lücken des $Z$ werchfells durch den Magen, dessen Serosa mit dem Periton. diaphr. umsäumt werden sollte, zu schließen und so die Entstehung eines Prolapses abdomineller Organe in die Brusthöhle zu verhüten. Sein Vorschlag entstammt der Frühperiode der "Chirurgie im unteren Brustraum".

Ausgiebige Entfernung der nach außen deckenden Brustwand ist eine Forderung für die in ihr Gebiet fallenden Operationen, auf deren Berechtigung, bzw. Notwendigkeit ich bereits früher hingewiesen habe. Indem ich ihr auch in diesem Falle nachkam, bot sich mir Gelegenheit, die Zweckmäßigkeit nach einer ganz neuen Richtung hin kennen zu lernen: Der a usgiebigen $\mathrm{Rip}$ penresektion ist es $z u$ verdanken, $d a B$ der groBe $Z$ werchfelldefekt sich unschwer durch die $\mathrm{Naht}$ schlieBen lie $B$.

Nach Entfernung der vorderen knöchernen Teile der 8.-Io. Rippe lassen sich ihre knorpeligen, zu einer Leiste vereinigten Enden bequem nach hinten drängen; sie folgen dem Zuge des $Z$ werchfells ebenso, wie das Ende der falschen Rippen, an dem die Portio costalis des Diaphragma mit ihren Zacken inseriert.

So läßt sich, wie ich mich auch an der Leiche überzeugte, eine erhebliche Verkleinerung in der Horizontalebene des unteren Brustraums erweisen. Sie ermöglichte den Verschluß der außer-

I) v, Hor o c k. Allg. Wiener med. Ztg. T884.

2) Groß, Erfahrungen uber Pleura- und Lungenchir. Beiträge zur klin. Chirurgie Bd. 24. 
gewöhnlich großen Zwerchfellsöffnung in unserem Falle, wenn ich mir auch nicht verhehle, daß ihre Lage in schräger Richtung und die leicht herzustellende Ovalform die Vornahme der Naht wesentlich erleichterten. Weiter kam noch als unterstützendes Moment hinzu, daß der Sinus diaphragmaticus verödet war, und durch seine $W$ iedereröffnung gewissermaßen ein Teil der Zwerchfellsfläche frei wurde; da bei der Untersuchung der Kranke keine Störung in der Bewegungsexkursion des linken Brustkorbes sich darbot, ist anzunehmen, daß der Ausfall des dem Sinus entsprechenden Teils ausgeglichen wurde, wahrscheinlich durch Dehnung des Muskels, soweit er nicht von der Geschwulst ergriffen war.

$\mathrm{Hahn}, \mathrm{Frey}, \mathrm{Marwed \textrm {e } ^ { 1 }}$ ), ähnlich v. Mikulicz, erreichten den Abschluß der Bauch- und Brusthöhle dadurch, daß sie die Lücken im $Z$ werchfell überbrückten, indem sie den medialen Wundrand mit der Brust- und Bauchwandmuskulatur vernähten.

Ich möchte annehmen, daß sich jeder Defekt durch die von mir angewandte Methode der ,Mobilisation der costalen Zwerchfellsinsertionen" bequem verschließenließe, in welcher Richtung er auch angelegt sei. Allerdings resultiert eine tiefe Einsattlung in Zwerchfellshöhe.

Das Zwerchfell in seiner Wölbung bietet zudem ein überaus plastisches Material.

Mit Ausnahme der Beobachtung von $\mathrm{Scalzi}{ }^{2}$ ), der nach Versorgung einer Stichverletzung ein Jahr später eine ausgebildete $Z$ werchfellshernie fand, ist anscheinend niemals, soweit ich feststellen kann, eine Insuffizienz der Zwerchfellsnaht weder früh, noch im weiteren Verlauf eingetreten.

A priori wäre man geneigt, gerade am Zwerchfell mit seinen ausgiebigen Bewegungen ungünstige Verhältnisse für die Sutur zu erwarten.

Verwerte ich die Lehre der bisherigen Erfahrungen und weiter speziell die meiner letzten Beobachtung, so bin ich geneigt, gerade das Gegenteil anzunehmen. Allerdings stehe ich noch immer unter dem Eindrucke der täglich immer wieder beim Verbinden kon-

I) $\mathrm{M}$ a r wed e bei $\mathrm{Amburger}$.

2) Sca $1 \mathrm{z}$. Gaz. med. ital.-lomb. I88I. 
trollierten Feststellung, daß in weiterer Umgrenzung der in ganzer Ausdehnung vorliegenden Zwerchfellsnaht fast gar keine Bewegungen des Zwerchfells hervortraten.

Es befand sich im Zustand der Ruhe, den ich mir nicht allein erklären kann durch Spannung des verkleinerten Muskels, da es sich eindrücken ließ, weiter auch nicht durch die ausschließliche Wirkung des äußeren Luftdruckes, des Pneumothorax, den wir nach Eröffnung der Wunde beim Verbandwechsel herstellten; bezüglich dieser Beurteilung des letzteren darf ich mich auf früher gesammelte (1. o.) und neuere Erfahrungen berufen, die beweisen, daß eine Beeinflussung der Zwerchfellsbewegung durch den äußeren Luftdruck nicht stattfindet, jedenfalls nicht in einem für das Auge erkennbaren Umfange.

Eher bin ich geneigt, für diesen der angelegten Naht so günstigen Zustand der Ruhe, den $\mathrm{O}^{\prime} \mathrm{D}$ w y e $\mathrm{r}^{\mathbf{1}}$ ) und S c a $1 \mathrm{z}$ i durch Bindeneinwicklung der Thorax herbeiführen wollten, die postoperative Infektion des unteren Brustraumes, die auch die Pleura diaphragmatica betraf, mit verantwortlich $\mathrm{zu}$ machen, weiter aber auch vielleicht die brüske mechanische Reizung des Zwerchfells durch die Naht, wie die sonstigen Manipulationen am Muskel. Sein besprochenes Verhalten wäre demnach z.T. als reflektorischer Akt aufzufassen.

Inwieweit die hierdurch bedingte mangelhafte ,Lüftung“ der Lunge für die Ausbildung der Pneumonie im Unterlappen förderlich war, sei dahingestellt. Wie ja anderweitige Erfahrungen ohne Eingriff am Zwerchfell lehren, genügt das Arbeiten an der Lunge, speziell resezierende Operationen mit weit sich erstreckender blutiger Infiltration, solche Operationspneumonien hervorzurufen.

I) O'D w yer. Med. Record I 889 . 